Check for updates

Cite this: RSC Adv., 2017, 7, 35504

Received 1st June 2017 Accepted 10th July 2017

DOI: 10.1039/c7ra06133a

rsc.li/rsc-advances

\title{
Dynamics of an amorphous pharmacologically active compound - diazepam: a QENS study combined with molecular dynamics simulations $\uparrow$
}

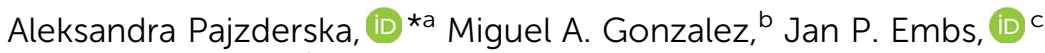 \\ Jadwiga Mielcarek ${ }^{d}$ and Jan W. Wąsicki ${ }^{\text {ae }}$
}

\begin{abstract}
The dynamics of methyl groups in crystalline and amorphous diazepam were investigated by a combination of molecular dynamics simulations and quasielastic neutron spectroscopy methods. The reorientation of the methyl group with a single correlation time was detected in crystalline diazepam. On the other hand, methyl group rotations in the amorphous sample cannot be represented by a single relaxation time and a distribution of correlation times approximated by a log-normal function is needed to explain both the simulation and neutron results. Additionally, MD simulations showed that the intermolecular part of the interactions due to the different environment around each methyl group is responsible for the distribution of correlation times observed experimentally.
\end{abstract}

\section{Introduction}

The study of amorphous forms of pharmacological active substances is becoming increasingly important due to the fact that they are often less thermodynamically stable and can show higher bioavailability ${ }^{\mathbf{1 - 5}}$ than the corresponding crystalline forms. It is therefore of interest to evaluate the molecular dynamics of amorphous active pharmaceutical ingredients since it is believed that mobility is one of the main factors that govern their physical stability. ${ }^{6,7}$ Investigations of amorphous forms are still a challenge, although very different experimental methods can be applied for the physico-chemical and dynamical characterization, including thermal analysis, X-ray powder diffraction, Fourier transform infrared spectroscopy (FT-IR), solid-state nuclear magnetic resonance (ssNMR) and neutron spectroscopy. ${ }^{8}$ Moreover, the macroscopic properties of amorphous substances could depend on the dynamics of molecular fragments. ${ }^{9}$ Thus, the understanding of the dynamics of functional groups, including methyl groups, is of general interest in drug design.

Diazepam belongs to the family of benzodiazepine derivatives, a group of medicaments having a wide spectrum of activity along four main lines: antianxiety, sedative and sleep-

\footnotetext{
${ }^{a}$ A. Mickiewicz University, Faculty of Physics, Umultowska 85, Poznan, Poland. E-mail: apajzder@amu.edu.pl

${ }^{b}$ Institute Laue Langevin, 71 Avenue des Martyrs, Grenoble, France

${ }^{c}$ Laboratory for Neutron Scattering and Imaging, Paul Scherrer Institut, 5232 Villigen, Switzerland

${ }^{d}$ Poznan University of Medical Sciences, Poznan, Poland

${ }^{e}$ The NanoBioMedical Centre, Umultowska 85, Poznan, Poland

$\dagger$ Electronic supplementary information (ESI) available. See DOI: 10.1039/c7ra06133a
}

inducing, anticonvulsive and reducing muscle tension. ${ }^{\mathbf{1 0}}$ The molecule is built of a condensed benzodiazepine ring and a chlorobenzene ring, with a methyl group attached to the nitrogen atom at position 1 in the benzodiazepine ring (Fig. 1).

The diazepam molecule contains only one methyl group and therefore, it is an excellent system to study the role of intra- and intermolecular interactions in determining the properties of pharmaceuticals in either crystalline or amorphous forms. In the past, we have studied the physical properties of crystalline diazepam by calorimetric, infrared absorption and nuclear magnetic resonance (NMR) methods. The investigation of its dynamics was complemented by a density functional theory (DFT) study of the vibrational frequencies and infrared intensities and the calculation of steric hindrances. The results indicate the occurrence of reorientation jumps of the $\mathrm{CH}_{3}$ group with a threefold barrier of height about $8 \mathrm{~kJ} \mathrm{~mol}^{-1},{ }^{11}$ which is typical value for reorientation of methyl groups. ${ }^{12-15}$ Later we focused on the amorphous sample,

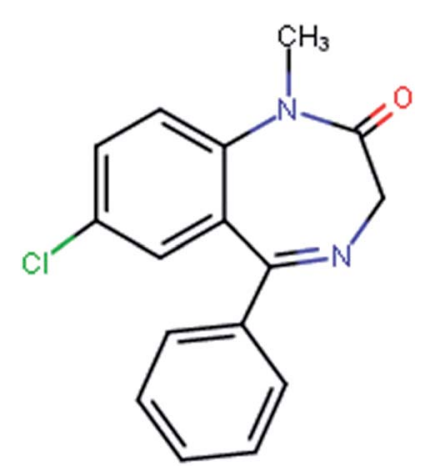

Fig. 1 Sketch of a diazepam molecule. 
obtained by the melting and quench-cooling method and used NMR and atom-atom potential energy calculations to determine the distribution of energy barriers for the reorientation of methyl groups, finding that there is a distribution of energy barriers varying from $\sim 2$ to $\sim 13 \mathrm{~kJ} \mathrm{~mol}^{-1}$. ${ }^{16}$ These calculations permitted also a separation of energy contributions coming from intra- and from inter-molecular interactions, with the former suggesting the possibility of a modification of the conformation of the diazepam molecule in the amorphous phase.

The drawback of atom-atom potential calculations is that they are performed under the static approximation, i.e. assuming that the neighbouring molecules are motionless. Nevertheless, the agreement between the energy barrier distribution obtained in this way for diazepam with that obtained from NMR measurements implies that the approximation is reasonably satisfactory.

However, in order to analyse the dynamics of amorphous diazepam in more detail and get further insight into the dynamical behaviour of this compound, in this paper we apply the molecular dynamics (MD) method to obtain and compare the correlation times of methyl group reorientations in crystalline and amorphous diazepam. In this way, we take into account the intraand intermolecular interactions and their modulation caused by the reorientation of methyl groups belonging to neighbouring molecules. The calculated correlation times are confronted with those measured for crystalline and amorphous diazepam using quasielastic neutron scattering (QENS). MD simulations permit the analysis of molecular motions whose time scale is comparable with that of QENS results and allow to trace the behaviour of individual methyl groups. Both methods are especially adequate for this kind of study, since they operate over essentially the same time and length scales, ideally complementing each other. The usefulness of such a combined approach resides in the direct confrontation of experimental and theoretical results. This allows us, on one hand, to validate the potential used in the MD simulations and, on the other hand, to use the MD trajectories as a guide for interpreting the experimental data and model the dynamical properties of the samples studied.

\section{Experimental}

\subsection{Sample}

Crystalline diazepam (7-chloro-1,3-dihydro-1-methyl-5-phenyl$2 \mathrm{H}$-1,4-benzodiazepin-2-one) was purchased in hermetically sealed containers from GlaxoSmithKline Pharmaceuticals S.A., Poznań. The quality of the sample was checked and described in our previous paper. ${ }^{11}$

Amorphous diazepam was prepared by melt-quench cooling methods, i.e. the sample was heated at $c a .10 \mathrm{~K} \mathrm{~min}^{-1}$ up to 425 $\mathrm{K}$ ( $\sim 10$ degrees above the melting temperature), kept at this temperature for approximately $10 \mathrm{~min}$ and then the melt was cooled down to $200 \mathrm{~K}$ (with a cooling rate equal to $20 \mathrm{~K} \mathrm{~min}^{-1}$ ).

\subsection{Computational details}

Molecular Dynamics (MD) simulations of crystalline and amorphous diazepam were performed using DL_POLY
Classic. ${ }^{17}$ Crystalline diazepam was simulated using a supercell of dimensions $38.8 \times 40.0 \times 39.9 \AA^{3}$ constructed on the basis of X-ray diffraction (XRD) data ${ }^{18}$ and containing 180 diazepam molecules. An amorphous cell containing 200 molecules was constructed using the Amorphous Cell module built in the Materials Studio package. ${ }^{19}$

MD simulations were performed in the temperature range 200-300 K at constant NVT. The temperature was controlled using Berendsen's thermostat ${ }^{20}$ with a relaxation constant of 1 ps. Periodic boundary conditions were applied in all directions. We used the cvff force field. ${ }^{21}$ A cut-off distance of $19 \AA$ was applied for the van der Waals forces and the electrostatic interactions were treated using the Ewald summation method with the same cutoff in real space. In all cases a time step of $1 \mathrm{fs}$ was used and the systems were equilibrated during $1 \mathrm{~ns}$. The trajectory was saved every 1 ps for a total simulation time of 10 ns. The analysis of the trajectories was then performed using nMoldyn 3 (ref. 22) and self-written programs.

\section{Methods}

QENS measurements of crystalline and amorphous samples were done on the time-of-flight spectrometer FOCUS (PSI, SINQ). The sample was placed in a specially designed container consisting in a hermetically closed aluminum hollow cylinder (mass of sample $=0.89 \mathrm{~g}$ ). QENS spectra were taken in the $Q$ range $0.3-1.8 \AA^{-1}$ using a PG002 monochromator, giving an incoming neutron wavelength $\lambda_{0}=5.75 \AA$ and an energy resolution equal to $\sim 0.036 \mathrm{meV}$ (FWHM). First, the crystalline sample was measured at the following temperatures: $26 \mathrm{~K}, 100$ $\mathrm{K}, 150 \mathrm{~K}, 200 \mathrm{~K}, 250 \mathrm{~K}, 300 \mathrm{~K}$ and $350 \mathrm{~K}$. Then, it was heated up to $425 \mathrm{~K}$ and amorphous diazepam was prepared by meltquench cooling methods and measured at the same temperatures as the crystal. Powder diffraction spectra (which are measured simultaneously on FOCUS) allowed us to follow the phase transitions and the disappearance of Bragg peaks confirmed the amorphisation of the sample. The sample holder was weighted before and after the measurements to confirm that it was hermetic and no mass losses were detected. Additionally, an empty container and a vanadium sample were measured at room temperature to be used in the background and normalization procedures, respectively.

Raw data were treated with the DAVE package, ${ }^{23}$ which performs the standard corrections, including background subtraction and self-absorption correction. Then, using a vanadium spectrum as an elastic scattering standard the intensities were normalised in order to correct for different detector efficiencies. Finally, detectors where Bragg peaks influenced the collected spectra were removed from further analysis in order to focus only on the incoherent quasielastic neutron scattering.

\section{Results and discussion}

\subsection{Molecular dynamics simulations}

Fig. 2 shows two snapshots corresponding to the initial configuration of the crystalline and amorphous simulation boxes on which the MD calculations were performed. 

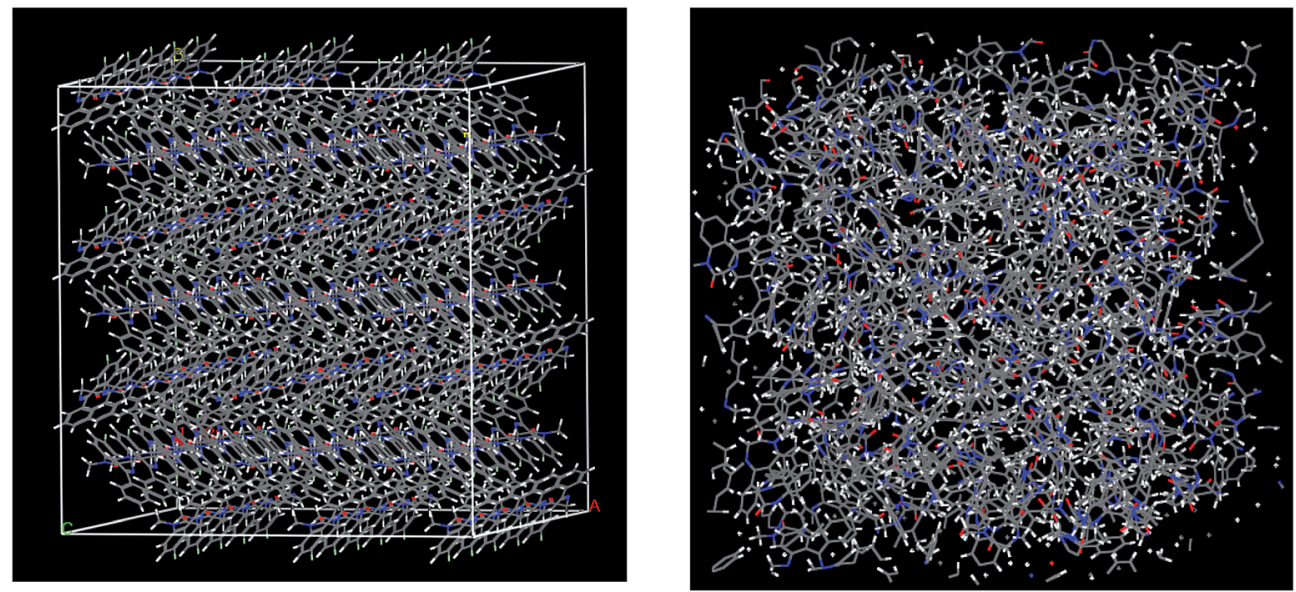

Fig. 2 Crystalline (left) and amorphous (right) simulation boxes used in the molecular dynamics simulations.

Crystalline diazepam. The $T_{1}$ relaxation time minimum observed by $\mathrm{NMR}^{\mathbf{1 1}}$ is caused by the reorientation of the methyl group from the diazepam molecule. Thus our first analysis consisted in following the behaviour of the $\mathrm{C}-\mathrm{H}$ bond vectors of all the methyl groups. Their reorientation can be characterized by the time dependence of $\theta(t)$, defined as the angle swept by the $\mathrm{C}-\mathrm{H}$ bond vector between $t=0$ and the time $t$ (see inset of Fig. 3). As clearly shown by the figure, methyl groups exhibit fast librations of small amplitude about a certain orientation, followed by nearly instantaneous jumps from one orientation to another. In other words, methyl groups rotate between three well defined positions, performing $120^{\circ}$ jumps between the orientations labeled as 0,120 and $-120^{\circ}$. The methyl dynamics can be described in a more quantitative way using the following time angular correlation function:

$$
\operatorname{ACF}(t)=\left\langle\vec{r}_{\mathrm{CH}}\left(t_{0}\right) \cdot \vec{r}_{\mathrm{CH}}\left(t_{0}+t\right)\right\rangle
$$

where $\vec{r}_{\mathrm{CH}}\left(t_{0}+t\right)$ is the vector along one $\mathrm{C}-\mathrm{H}$ bond of a methyl group at time $t_{0}+t$ and the brackets indicate an average over all methyl groups in the system and possible origins $t_{0}$. The ACFs can be fitted using a single exponential function $(\operatorname{ACF}(t) \sim$ $\left.\exp \left(-t / \tau_{\mathrm{c}}\right)\right)$, where $\tau_{\mathrm{c}}$ is the correlation time. The angular correlation function (ACF) was calculated both as an average of all the methyl groups and for each individual methyl groups. The results obtained at $300 \mathrm{~K}$ are shown in Fig. 3.

Unsurprisingly, the behaviour of all the methyl groups in the crystal is very similar, and all of them show comparable relaxation times. With decreasing temperature, the methyl reorientation slows down as expected. The temperature dependence of the average correlation time is displayed in Fig. 4, which also shows the $\tau_{\mathrm{c}}$ dependence obtained using the activation parameters from the earlier NMR measurements and an Arrhenius relation (solid line) and those derived from QENS data (next section of this work). The three sets of correlation times are in excellent agreement, suggesting that the method of calculation, as well as the partial charges and force field employed to describe the dynamic properties of diazepam are a good choice and can be extended to the amorphous sample.

Amorphous diazepam. Therefore, analogous calculations were performed for amorphous diazepam. Fig. 5 is the counterpart of Fig. 3, showing the evolution during the trajectory of $\theta(t)$ for 5 representative molecules and their corresponding ACFs. As before the data can be understood in terms of fast librations of the methyl groups about the equilibrium position with occasional 120 degrees jumps. However, in the amorphous system different molecules can show very disparate behaviour. While the jumping frequency for some methyl groups is very high, others show only occasional jumps and can remain 'locked' in a particular position during quite long times (up to a few hundreds of ps, as shown in Fig. 5).

As before, the ACF of each methyl group was calculated individually and fitted by a single exponential function from which the correlation times were extracted. The shortest correlation time found is $0.5 \mathrm{ps}$, while the longest is $\sim 50 \mathrm{ps}$ (at $300 \mathrm{~K}$ ) or $\sim 200 \mathrm{ps}$ (lower temperatures), indicating a very broad distribution of relaxation times. In order to characterize such a distribution, we show in Fig. 6 the histogram obtained by representing the number of molecules (or methyl groups) characterised by a given correlation time $\tau_{\mathrm{c}}$. At $350 \mathrm{~K}$ this distribution is highly non-symmetric and shows a single maximum at $\tau_{\mathrm{c}} \approx 2 \mathrm{ps}$ followed by a long tail extending to very long times. With decreasing temperature, the reorientation slows down (so the maxima are shifted towards longer times) and the distribution of correlation times extends, indicating that an increased number of methyl groups is characterised by longer correlation times.

The distributions shown in Fig. 6 can be well described by the log-normal function:

$$
g_{j}\left(\tau_{\mathrm{c}, j}\right)=\frac{1}{\sigma \sqrt{2 \pi}} \exp \left[-\frac{1}{2 \sigma^{2}} \ln ^{2}\left(\frac{\tau_{\mathrm{c}, j}}{\tau_{\mathrm{c}, \max }}\right)\right],
$$

where $\sigma$ is the standard deviation of the distribution and $\tau_{\mathrm{c} \text {,max }}$ the correlation time corresponding to the maximum of the distribution. 


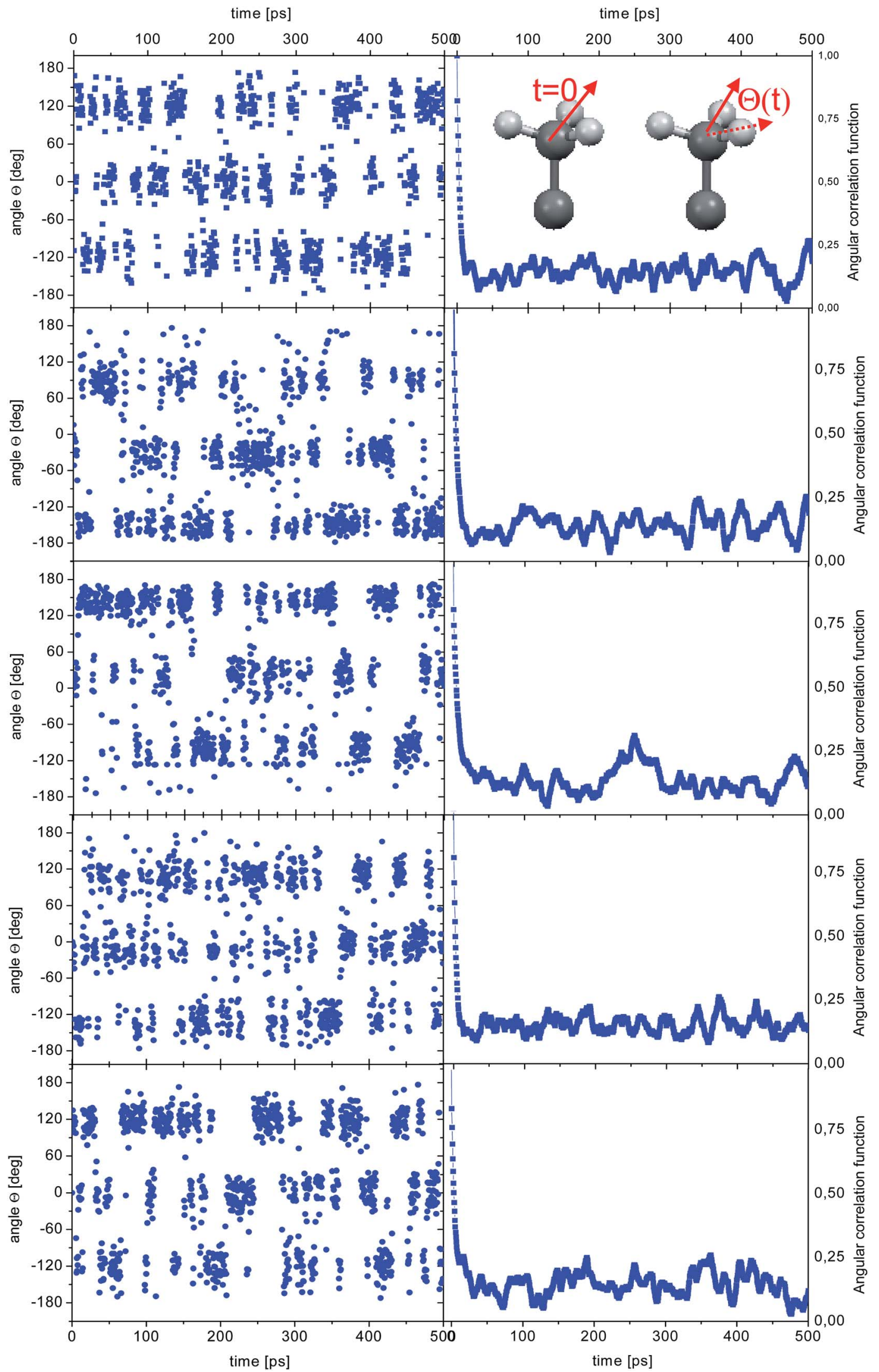

Fig. 3 Time dependence of the angle $\theta(t)$ for the reorientation of selected methyl groups in the simulation of crystalline diazepam (left) and corresponding ACF (right). The inset shows an sketch of a methyl group with the definition of the angle $\theta$. 


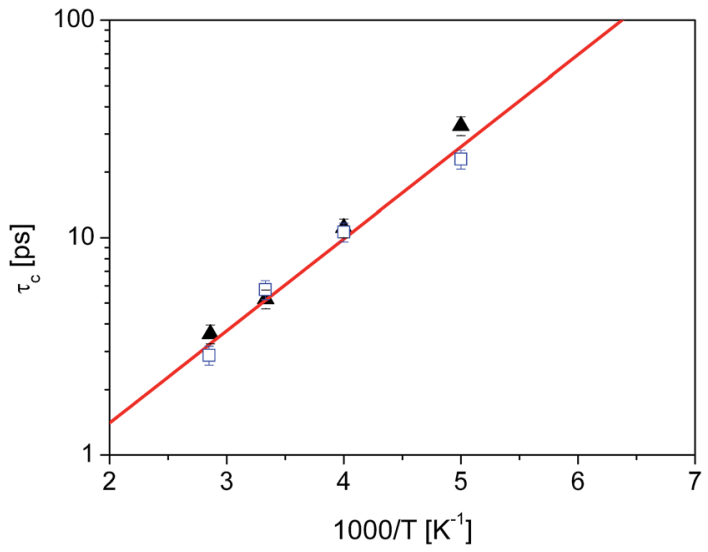

Fig. 4 Temperature dependence of the correlation time for methyl reorientation in crystalline diazepam: $\square$ QENS data $\boldsymbol{\Delta}$ MD simulations, solid line: NMR data. ${ }^{16}$

\subsection{Quasielastic neutron scattering}

As mentioned above, apart from the QENS spectra, during the same measurements also the neutron diffraction patterns were recorded. They are shown in Fig. $1 \mathrm{~S} \dagger$ for both crystalline and amorphous diazepam measured at room temperature. As expected, the crystal exhibits well pronounced diffraction peaks appearing at scattering angles that agree with those generated on the basis of the known crystallographic structure. For the amorphous sample, no diffraction peaks are observed, and only a very broad peak (a halo) is seen with a maximum at about 50 degrees, confirming that the quench-cooling process succeeded in producing an amorphous sample.

Crystalline diazepam. The spectra of all crystalline samples were fitted with the following expression (convoluted with the resolution function $R(Q, \omega))$ :

$$
S(Q, \omega)=A_{0}(Q) \delta(\omega)+\left(1-A_{0}(Q)\right) L(\omega)+B(Q, \omega),
$$

where $A_{0}(Q)$ is the elastic incoherent structure factor (EISF), $L(\omega)=\frac{1}{\pi} \frac{\Gamma}{\omega^{2}+\Gamma^{2}}$ is a Lorentz function of half-width at half-maximum $\Gamma$ and $B(Q, \omega)$ describes the linear background. They were fitted using the STR_FIT program in Lamp ${ }^{24}$ which enables numerical convolution with the instrumental resolution function $R(Q, \omega)$ determined from a measurement of a vanadium standard sample.

Two important parameters are extracted from the fit: the correlation time (inversely proportional to the half-width of the Lorentz function $\Gamma$ ) and $A_{0}(Q)$, which provides information about the geometry of the dynamic process. Thus, this phenomenological fit permits us to determine the EISF in a model independent way. Assuming jumps of methyl groups between three equidistant sites on a circle of radius $r$, the corresponding EISF can be written as: ${ }^{25}$

$$
A_{0}(Q)=\frac{1}{3}\left[1+2 j_{0}(Q r \sqrt{3})\right]
$$

While all atoms in the sample contribute to the EISF, the contribution from the hydrogen atoms represents more than $90 \%$ of the total scattering, so the contribution from other atoms (nitrogen, carbon, oxygen, and chlorine) can be neglected. Furthermore in the crystalline sample we can assume that only the methyl groups move within the time scale given by the finite resolution of the spectrometer. Therefore the measured elastic incoherent structure factor (EISF) can be written as

$$
\mathrm{EISF}=c+(1-c) \cdot A_{0}(Q)
$$

where $c=0.77$ is the fraction of immobile hydrogen atoms in the experimental time scale. The measured EISF obtained from the fit of the QENS spectra using eqn (3) is displayed in Fig. 7, together with the theoretical lines corresponding to eqn (5). The perfect agreement between theoretical expectation and the experimentally derived EISFs indicate that the only motion observed in the QENS experiments is effectively the rotation of the methyl groups between three equivalent positions. For this model the half-width $\Gamma$ obtained from the fitting of the QENS spectra is related to the correlation time $\tau_{\mathrm{c}}$ by the expression $\tau_{\mathrm{c}}=3 \hbar /(2 \Gamma){ }^{25}$ As shown before (Fig. 4), the correlation times obtained at different temperatures from the QENS data are also in very good agreement with the NMR data.

In the range $250-350 \mathrm{~K}$, the EISF obtained does not depend on temperature (Fig. 7) and is perfectly described by the model assuming the reorientation of one methyl group. Therefore all the spectra were re-fitted using this specific model corresponding to jumps between three positions:

$$
\begin{aligned}
S(Q, \omega)= & \exp \left(-\frac{u^{2} Q^{2}}{3}\right)\left\{\left[c+(1-c) A_{0}(Q)\right] \delta(\omega)\right. \\
& \left.+(1-c)\left(1-A_{0}\right) \frac{1}{\pi} \frac{\Gamma}{\omega^{2}+\Gamma^{2}}\right\}
\end{aligned}
$$

where $\exp \left(-\mathrm{u}^{2} \mathrm{Q}^{2} / 3\right)$ is the Debye-Waller factor. Again this model describes very well the experimental data, as shown in Fig. 8, but now using only two parameters, $r$ and $\Gamma$, as the $Q$ dependence of $A_{0}(Q)$ is now given by eqn (6).

Amorphous diazepam. QENS spectra recorded for amorphous diazepam differ from those of the crystalline form. Initial attempts were made to describe the spectra using eqn (3) and (6), but they did not provide any satisfactory agreement (see Fig. 9). This can be understood by referring to earlier studies, ${ }^{\mathbf{1 6}}$ as well as to the MD simulation results previously presented, which indicate that the reorientation of methyl groups in the amorphous phase cannot be described by a single correlation time. The QENS data confirm this observation, as if the reorientation of methyl groups were characterised by a single correlation time $\tau_{\mathrm{c}}$, the quasielastic broadening could be described by a function containing a single Lorentz function. Instead we find that the description of the experimental curves requires the use of several Lorentz functions. Therefore the following model was chosen to describe the experimental results: 


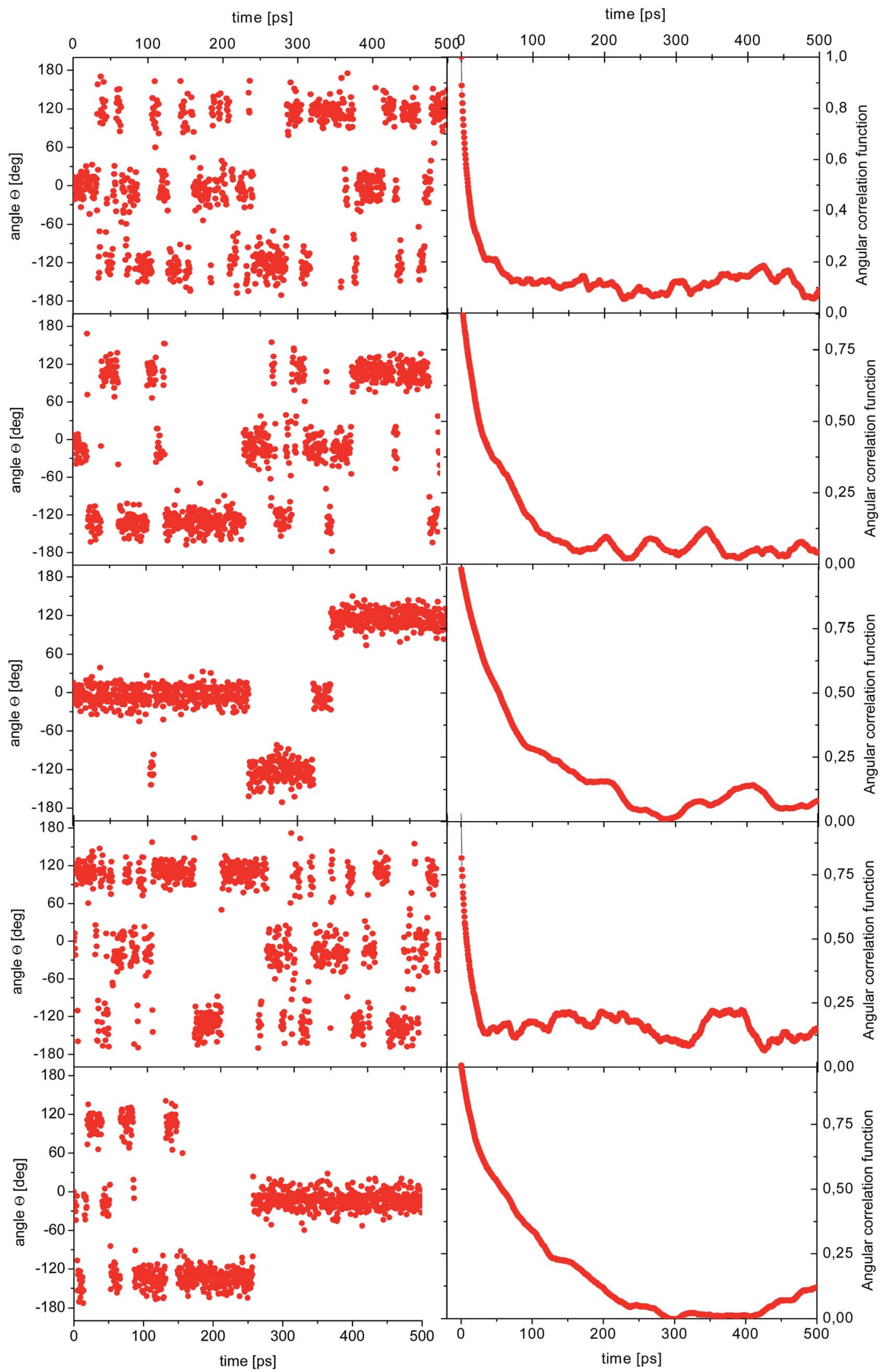

Fig. 5 Time dependence of the angle $\theta(t)$ for the reorientation of selected methyl groups in the simulation of amorphous diazepam (left) and corresponding ACFs (right). 

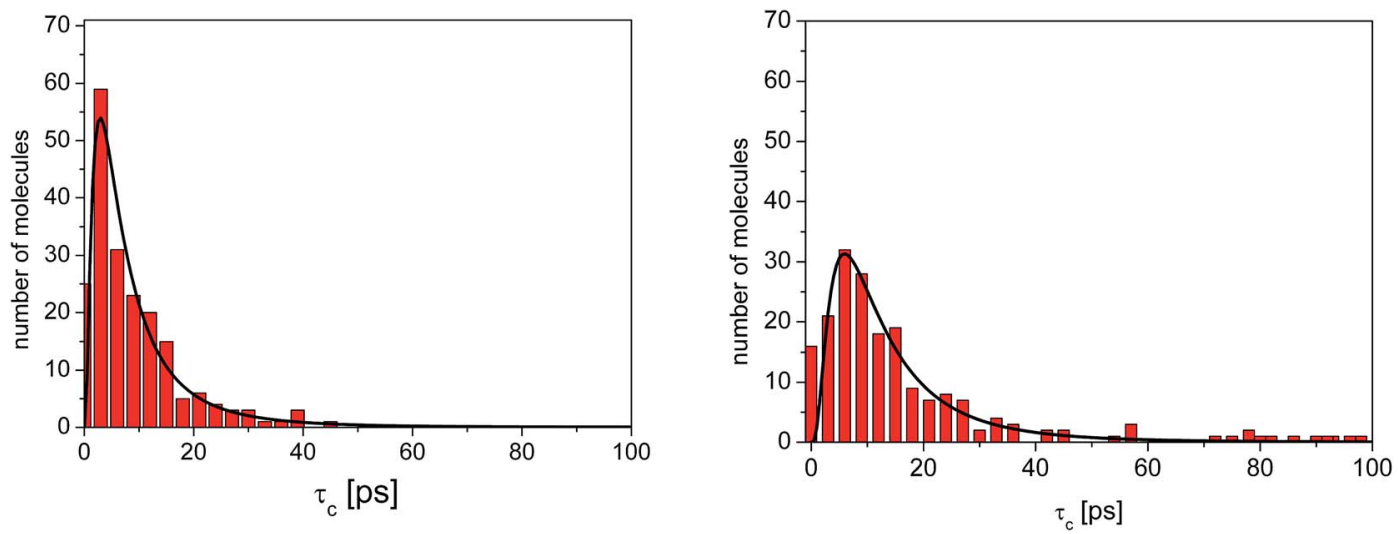

Fig. 6 Distribution of correlation times for the reorientation of methyl groups in amorphous diazepam extracted from MD simulations (rectangles) at $300 \mathrm{~K}$ (left) and $250 \mathrm{~K}$ (right). The solid lines correspond to the curves obtained by fitting this distribution with a log-normal function (eqn (2)).

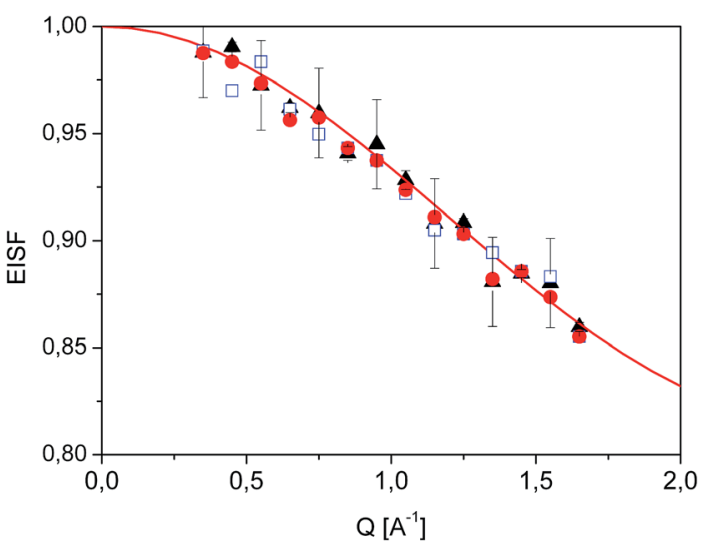

Fig. 7 Experimental EISF extracted from QENS data at $T=350 \mathrm{~K}(\mathbf{\Delta})$, $300 \mathrm{~K}(\bullet)$ and $250 \mathrm{~K}(\square)$. The solid line shows the expected EISF corresponding to a methyl rotation between three equivalent positions while the other atoms of the diazepam molecule remain immobile (eqn (5)).

$$
\begin{aligned}
S(Q, \omega)= & \exp \left(-\frac{u^{2} Q^{2}}{3}\right)\left\{\left[c+(1-c) A_{0}(Q)\right] \delta(\omega)\right. \\
& \left.+(1-c)\left(1-A_{0}\right) \sum_{j=1}^{L} g_{j} \frac{1}{\pi} \frac{\Gamma_{j}}{\omega^{2}+\Gamma_{j}^{2}}\right\}
\end{aligned}
$$

where:

$$
g_{j}=g\left(\ln \Gamma_{j}\right)=\frac{1}{\sigma \sqrt{2 \pi}} \exp \left[-\frac{1}{2 \sigma^{2}} \ln ^{2}\left(\frac{\Gamma_{j}}{\Gamma_{i}}\right)\right]
$$

This model is based on the rotation distribution model ${ }^{26}$ that considers a distribution of jumping rates and has been applied in polymers, ${ }^{27,28}$ in low molecular-weight glasses ${ }^{29,30}$ or in glassforming liquids in soft confinement. ${ }^{31}$ For each jumping distance, instead of a single $\Gamma_{i}$ value, a distribution of HWHMs is used. The distribution is represented by $L$ values of the HWHM $\left(\Gamma_{j}\right)$ with associated weights $g_{j}$ taken from a log-normal distribution of standard deviation $\sigma$ and normalized such that $\sum_{j=1}^{L} g_{j}=1$. The $\Gamma_{j}$ are chosen equally spaced on a logarithmic scale in the range $\exp \left(-\sigma \sqrt{-2 \ln A_{\min }}\right), \exp \left(\sigma \sqrt{-2 \ln A_{\min }}\right)$, where $A_{\min }$ is the cut-off chosen for the value of the distribution function with respect to its maximum. Here we used $L=21$ and $A_{\text {min }}=0.1$.

Fig. 9 presents an example of the QENS spectra of amorphous diazepam measured at $250 \mathrm{~K}$ and several $Q$ values together with the results of the fits obtained with eqn (7). As mentioned above, the half-width $\Gamma_{j}$ obtained from the fitting of the QENS spectra is directly related to the correlation time $\tau_{\mathrm{c}}$. As each Lorentz function of width $\Gamma_{j}$ represents a contribution described by the weight $g_{j}$, it is possible to obtain a distribution of correlation times. They are shown in Fig. 10 together with the distribution obtained from the MD simulations (see Fig. 6). Both present similar characteristics, although the experimental one cannot be estimated beyond a few tens of ps due to the time-window of FOCUS spectrometer.

\subsection{Analysis of intra- and intermolecular interactions}

In order to determine the origin of the very different behaviour in crystalline and amorphous diazepam, the MD trajectories were used to probe the intra- and intermolecular structure by means of the partial and total radial distribution functions (RDF). The latter is computed as:

$$
g(r)=\frac{\sum_{\alpha, \beta}^{n} c_{\alpha} c_{\beta} w_{\alpha} w_{\beta} g_{\alpha \beta}(r)}{\sum_{\alpha, \beta}^{n} c_{\alpha} c_{\beta} w_{\alpha} w_{\beta}}
$$

And each partial RDF is given by:

$$
g_{\alpha \beta}(r)=\frac{\rho_{\alpha \beta}(r)}{\bar{\rho}_{\alpha \beta}},
$$




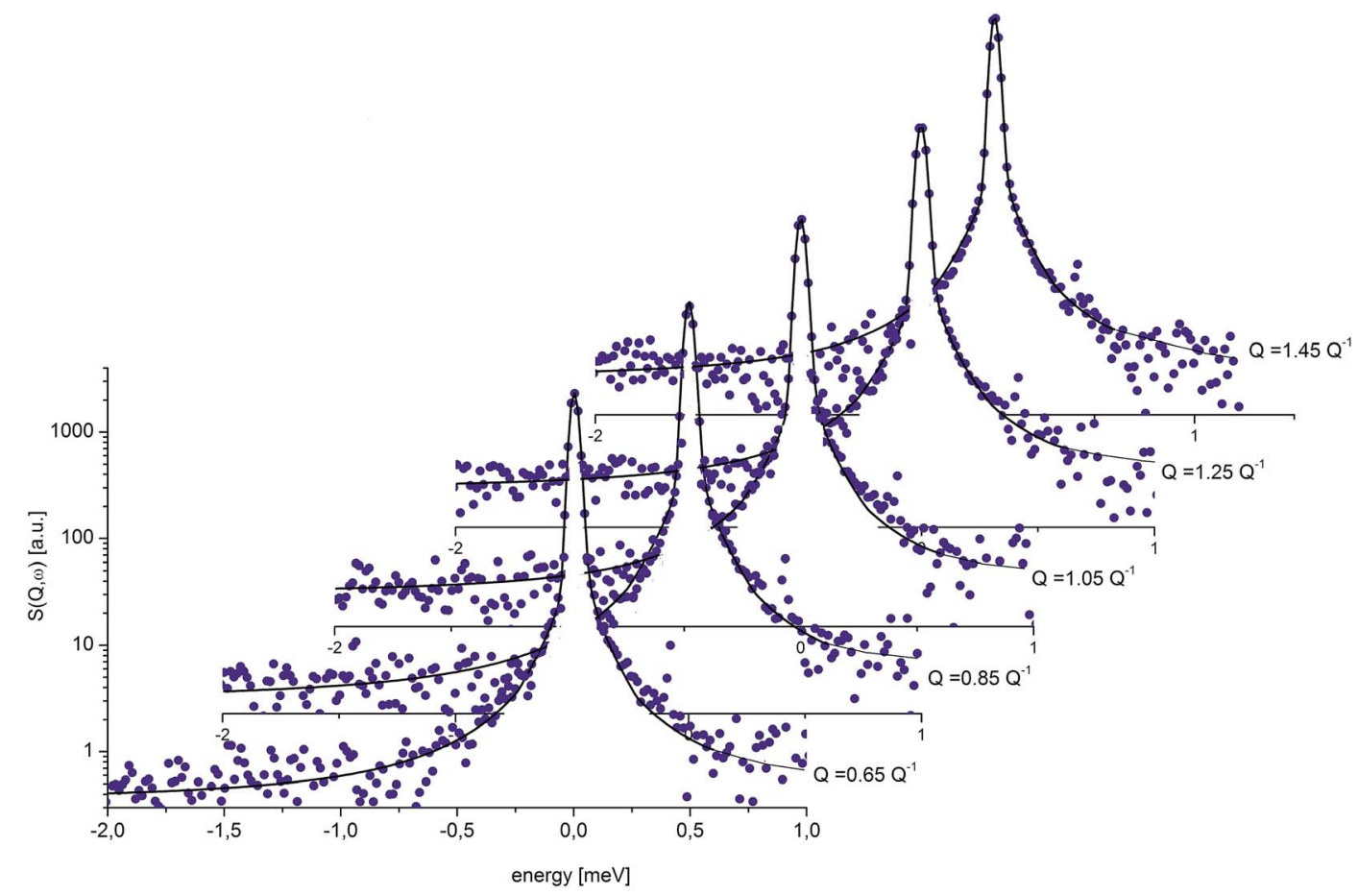

Fig. 8 QENS spectra for crystalline diazepam at $250 \mathrm{~K}$. The solid line shows the fits given by eqn (6).

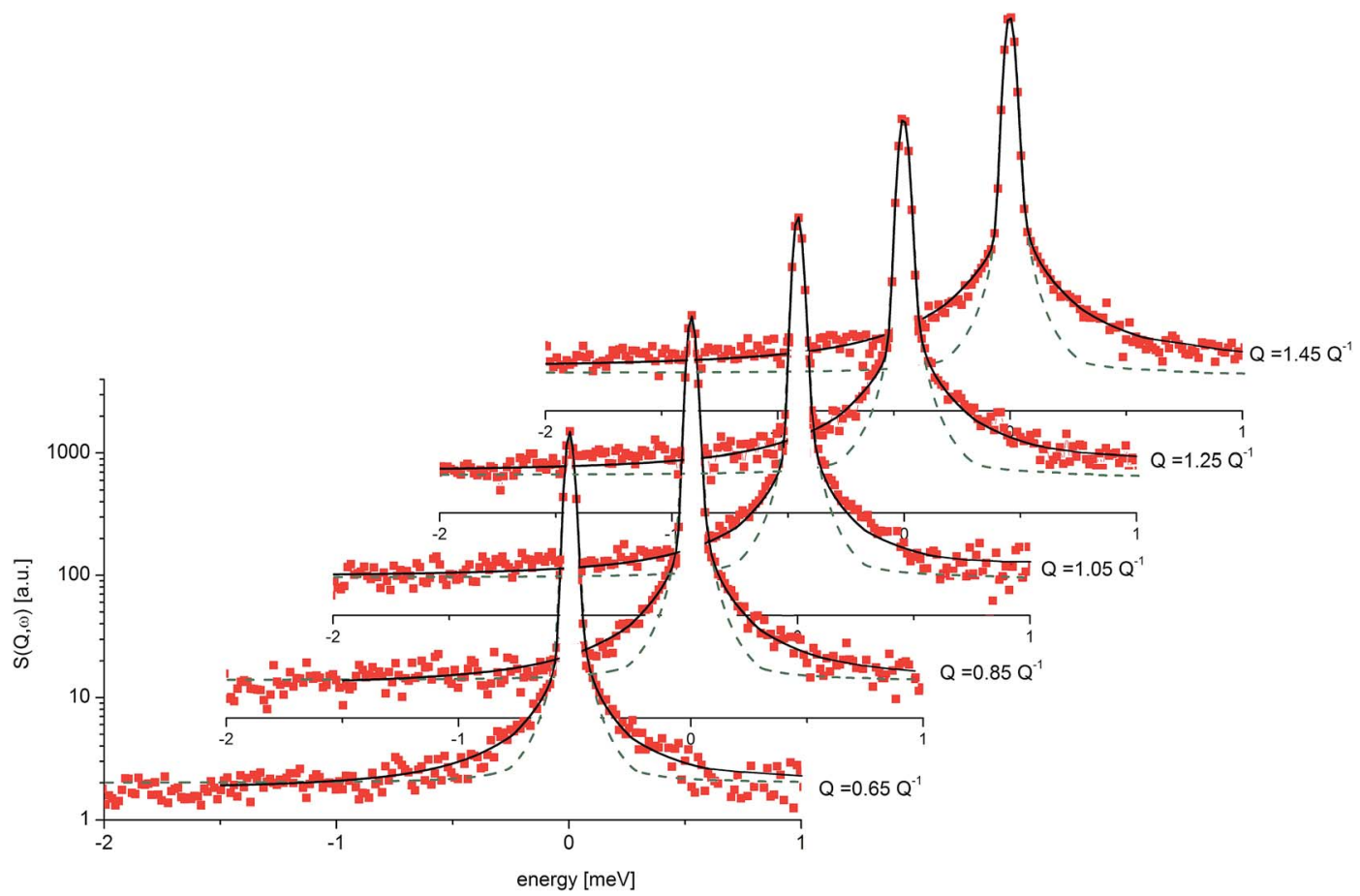

Fig. 9 QENS spectra for amorphous diazepam at $250 \mathrm{~K}$. The solid lines correspond to the fits obtained using eqn (7), while the dashed lines to those using eqn (6). The difference between both fits shows clearly that the model assuming only one correlation time (one single Lorentzian function) cannot describe well the experimental data.

where $\rho_{\alpha \beta}$ represents the local density of atoms of type $\beta$ in a shell of radius $r$ and thickness $d r$ centered at an atom of type $\alpha$ and $\bar{\rho}_{\alpha \beta}$ represents the average density over the whole simulation box, $c_{\alpha}$ and $c_{\beta}$ correspond to the concentration numbers of atoms of type $\alpha$ and $\beta$, respectively and $w_{\alpha}$ and $w_{\beta}$ to their respective weights in the total $\mathrm{RDF}$ (e.g. neutron scattering lengths or X-ray form factors when comparing with neutron or X-ray diffraction data, but here set to 1 for all the atoms). 

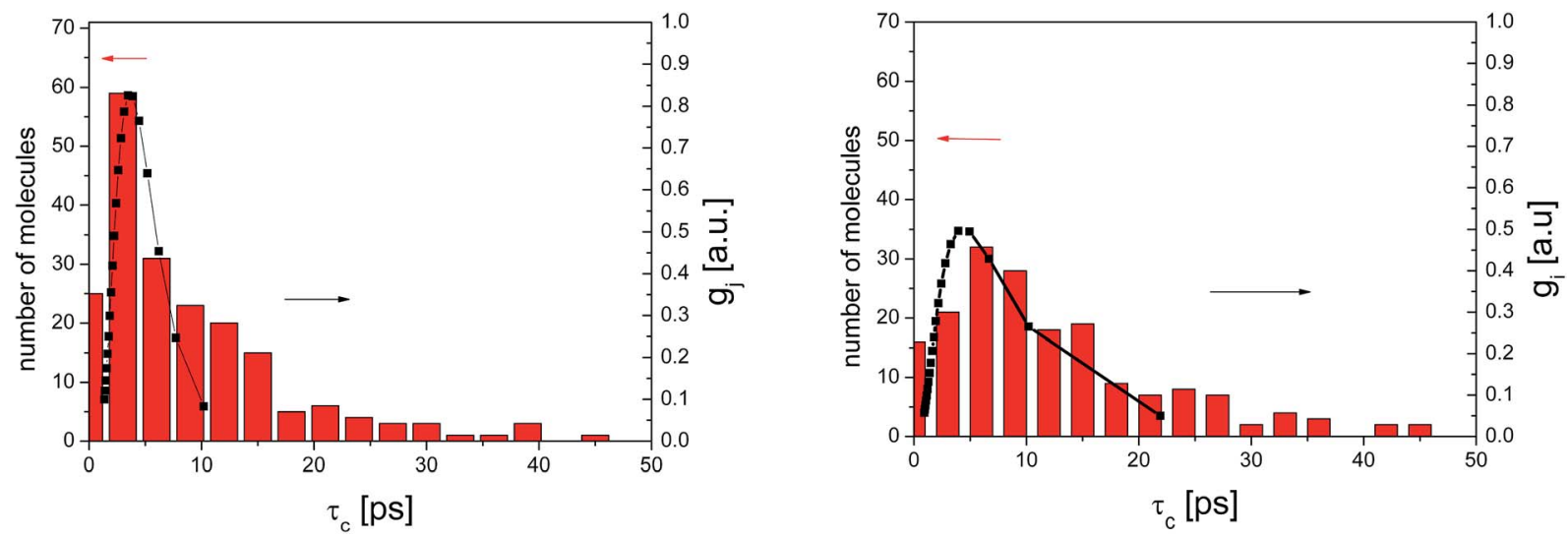

Fig. 10 Distribution of correlation times for amorphous diazepam as obtained from MD simulation (rectangles) and from the fit of QENS data at $300 \mathrm{~K}$ (left) and $250 \mathrm{~K}$ (right) with eqn (7) (solid lines).

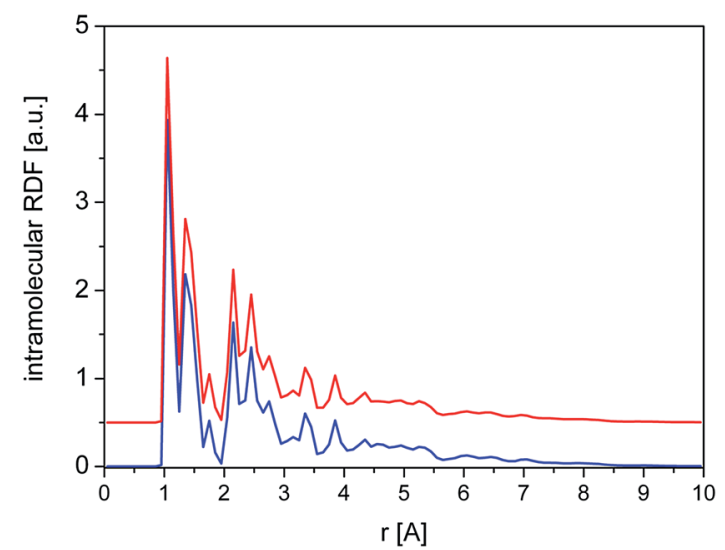

Fig. 11 Total intramolecular RDF calculated for crystalline (blue line) and amorphous (red line) cluster.

The total intramolecular RDF (Fig. 11) shows several wellresolved peaks and their positions and widths are very similar for crystalline and amorphous samples, clearly indicating that the molecular geometry is almost identical in both phases and reflecting the relative rigidity of the diazepam molecule.
This is further confirmed by a closer examination of the distribution of intramolecular distances (Fig. 12a) between the C of the methyl group and three reference atoms situated in each of the rings forming the molecule, i.e. $\mathrm{O}, \mathrm{Cl}$ and the $\mathrm{C}$ situated at the extreme of the phenyl ring. Again, the positions and width of those peaks are almost identical for both phases. Only the last RDF (peak at $\sim 8.5 \AA$ ) is slightly broader in the amorphous system, suggesting some additional flexibility of the phenyl ring compared to the crystal. We also calculated the equivalent RDFs for the hydrogen of the methyl groups, which show some additional structure (Fig. 12b), but there are no significative differences between crystal and amorphous that could explain the different dynamical behaviour of the methyl group in both phases.

The nature of the local environment has been determined by calculating the RDF individually for each methyl group, i.e.

$$
g_{\mathrm{H}_{i} \mathrm{X}}(r)=\frac{\left[\sum_{j \neq i}^{N_{\mathrm{m}}} \sum_{\alpha=1}^{N_{\mathrm{a}}} n_{\mathrm{H}_{i} \alpha_{j}}(r) / V_{\text {shell }}(r)\right]}{\left[3\left(N_{\mathrm{m}}-1\right) N_{\mathrm{a}} / V_{\mathrm{box}}\right]},
$$

where $\mathrm{H}_{i}$ represents the hydrogen atoms of the methyl group in molecule $i, N_{\mathrm{m}}$ is the number of diazepam molecules in the simulation box, $N_{\mathrm{a}}$ the number of atoms per molecule, $V_{\mathrm{box}}$ the
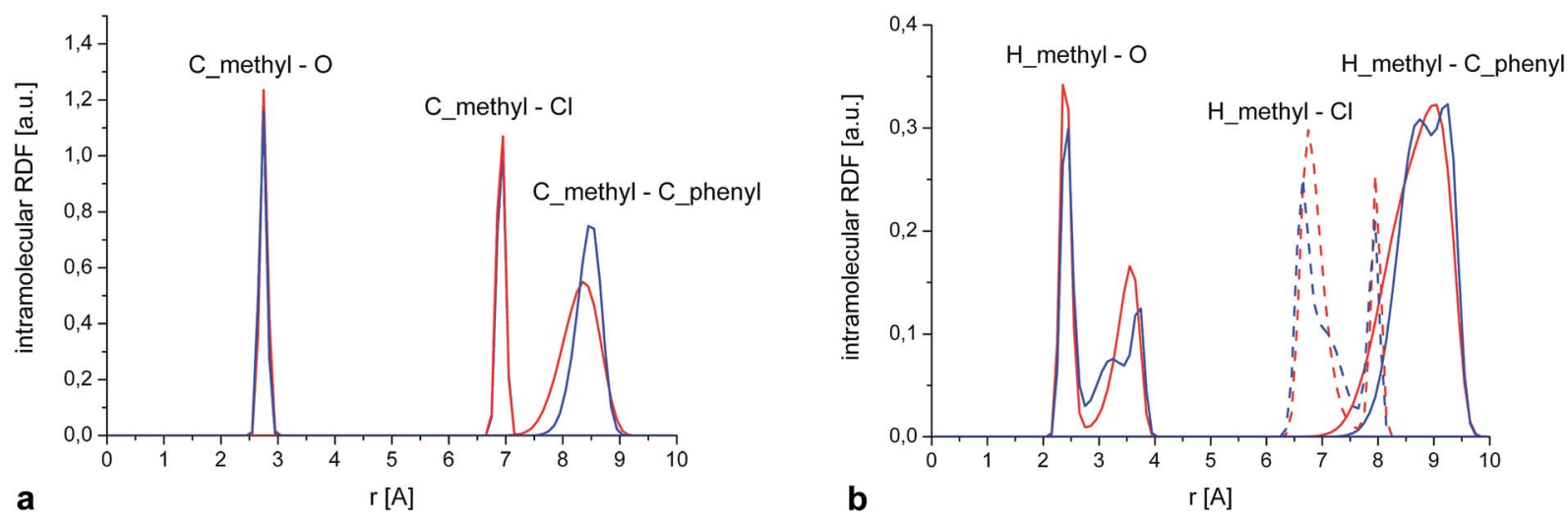

Fig. 12 (a) Partial intramolecular RDFs calculated for crystalline and amorphous samples showing the distance distribution between the $C$ of the methyl group and the extreme $\mathrm{C}$ of the phenyl ring, the oxygen (RDF scaled by a factor 20) and the chlorine atom (RDF scaled by a factor 3). (b) As (a), but replacing the methyl carbon by the methyl hydrogens. 

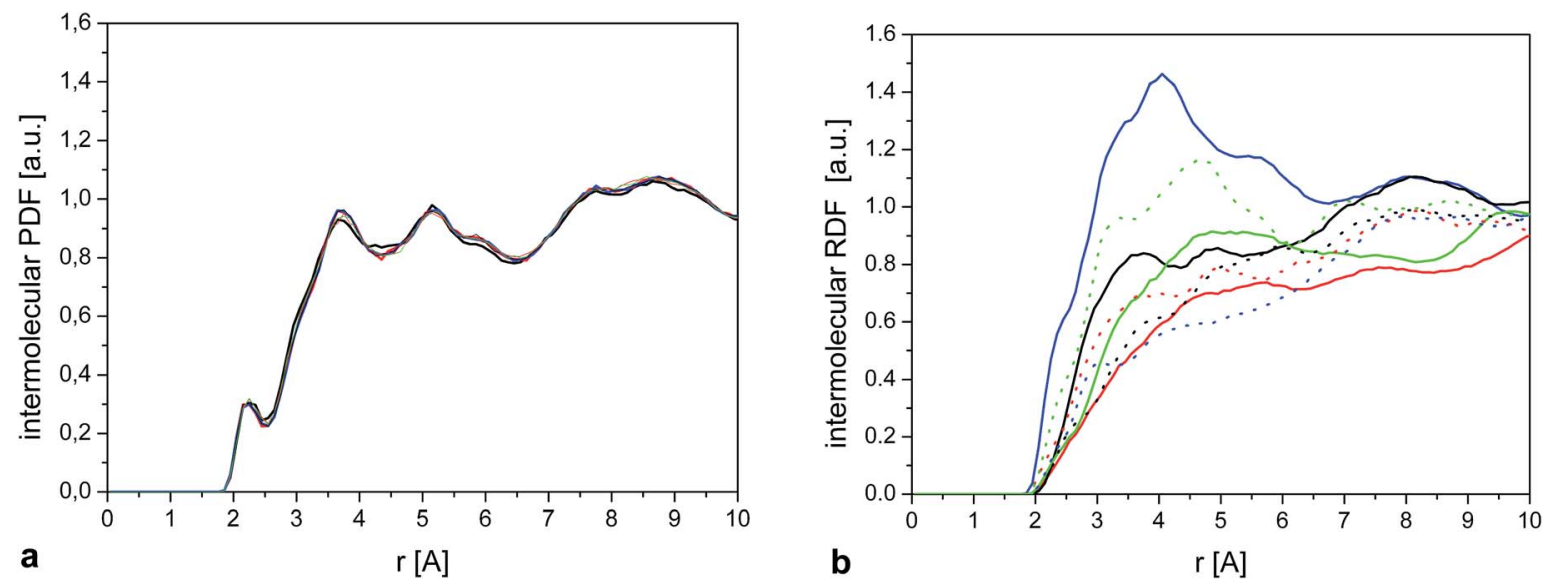

Fig. 13 (a) Intermolecular $\mathrm{H}_{i} \mathrm{X}$ RDF (eqn (9)) calculated for a single methyl group. The 3 curves show the result for 6 randomly selected molecules. (b) As (a) for 6 randomly selected molecules in the amorphous sample.

volume of the simulation box, $V_{\text {shell }}(r)$ the volume of an spherical shell of thickness $d r=0.1 \AA$ at a distance $r$, and $n_{\mathrm{H}_{i} \alpha_{j}}(r)$ corresponds to the number of atoms of any molecule $j \neq i$ situated at a distance in the range $r$ and $r+d r$ of any of the 3 hydrogens of the methyl group of molecule $i$.

In the case of the crystal (Fig. 13), we see that the environment around the methyl group is very similar for each molecule, as expected, explaining that the $\mathrm{CH}_{3}$ rotation can be described by a single correlation time. Also contributions from different atoms to RDF are very similar to each other (Fig. 2S $\dagger$ ).

On the other hand, we observe significant differences between the molecules in the amorphous system. Fig. 13b shows the intermolecular RDF for six selected molecules from the amorphous system and Fig. $2 \mathrm{~S} \dagger$ the separate contributions from different types of atoms for these molecules. The RDF around each methyl group in the amorphous phase is very different, so it comes as no surprise that they will also have different rotational correlation times.

It is tempting to try to link the individual correlation times previously obtained (e.g. Fig. 5) with the different steric hindrances exemplified by the RDFs shown above. Although we explored this path, we could not establish any obvious correlation. This is due to the fact that the effective rotational potential felt by a methyl group will emerge from the combination of all the intra- and inter-molecular interactions which have different magnitudes and ranges (torsional potential, van der Waals interactions with different values of $\sigma$, and electrostatic potential). Therefore the only conclusion that we can extract is that the variety of environments in the disordered phase of diazepam results in a distribution of correlation times that can be well represented by a log-normal function, as shown in Fig. 10.

\section{Conclusions}

In this paper, by combining molecular dynamics simulations and quasielastic neutron scattering we have investigated in detail the dynamics of methyl groups in crystalline and amorphous diazepam. Our results can be summarised as follows:
(1) Molecular dynamics simulations performed for crystalline diazepam confirmed the occurrence of reorientations of methyl groups and permitted the determination of the temperature dependence of their characteristic correlation times, which are in very good agreement with those obtained from NMR and QENS measurements.

(2) Molecular dynamics simulations revealed that the correlation times of reorientations of methyl groups in the amorphous sample cannot be characterized by a single correlation time, but by a distribution of correlation times approximated by a log-normal function.

(3) The QENS spectra obtained for the crystalline sample could be fitted assuming a simple model consisting of a $\delta$ function and one single Lorentzian function of width $\Gamma$.

(4) The spectra of amorphous diazepam are more complex and could be fitted by the modified rotation distribution model - a log-Gaussian model (following from MD simulations) that assumes a distribution of jumping rates, so instead of a single $I$ value, a distribution of widths is used.

(5) MD simulations showed that the distribution of correlation times observed experimentally are due to intermolecular effects and the variety of local environments around the methyl group that one can have in the amorphous sample.

In summary, molecular dynamics simulations have allowed us to obtain a more detailed understanding of the dynamics of molecular fragments in crystalline and amorphous drugs, shedding new light on the origin of the distribution of correlation times in amorphous systems also observed in QENS experiments.

\section{Acknowledgements}

The work has been partially financed by the National Science Centre of Poland, Grant No. 2015/17/B/ST5/00104 and has been supported in part by PL-Grid Infrastructure. A. P. would like to thank the Institut Laue Langevin (France) for an invitation as a visiting scientist. This work is based on experiments performed at the Swiss spallation neutron source SINQ, Paul Scherrer Institute, Villigen, Switzerland. 


\section{References}

1 A. M. Thayer, Form and Function, Chem. Eng. News, 2007, 85, 17-30.

2 A. M. Thayer, Finding Solutions, Chem. Eng. News, 2010, 88, 13-18.

3 T. Einfalt, O. Planinsek and K. Hrovat, Methods of Amorphization and Investigation of the Amorphous State, Acta Pharm., 2013, 63, 305-334.

4 B. C. Hancock and G. Zografi, Characteristics and Significance of the Amorphous State in Pharmaceutical Systems, J. Pharm. Sci., 1997, 86, 1-12.

5 P. Srinarong, S. Kouwen, M. R. Visser, W. L. Hinrichs and H. W. Frijlink, Effect of Drug-carrier Interaction on the Dissolution Behavior of Solid Dispersion Tablets, Pharm. Dev. Technol., 2010, 15, 460-468.

6 A. Paudel, M. Geppi and G. van den Monter, Structural and Dynamic Properties of Amorphous Solid Dispersions: the Role of Solid State Nuclear Magnetic Resonance Spectroscopy and Relaxometry, J. Pharm. Sci., 2014, 103, 2635-2662.

7 S. Baghel, H. Cathcart and N. J. O'Reilly, Polymeric Amorphous Solid Dispersions: A Review of Amorphization, Crystallization, Stabilization, Solid-State Characterization, and Aqueous Solubilization of Biopharmaceutical Classification System Class II Drug, J. Pharm. Sci., 2016, 105, 2527-2544.

8 P. Gao, Amorphous Pharmaceutical Solids: Characterization, Stabilization, and Development of Marketable Formulations of Poorly Soluble Drugs with Improved Oral Absorption, Mol. Pharm., 2008, 5, 903-904.

9 For example: Y. Aso, S. Yoshioka and S. Kojima, Molecular Mobility-Based Estimation of the Crystallization Rates of Amorphous Nifedipine and Phenobarbital in poly(vinylpyrrolidone) Solid Dispersions, J. Pharm. Sci., 2004, 93, 384-391.

10 F. Qashu, T. H. Figueiredo, V. Aroniadou-Anderjaska, J. P. Apland and M. F. Braga, Diazepam Administration After Prolonged Status Epilepticus Reduces Neurodegeneration in the Amygdala but not in the Hippocampus During Epileptogenesis, Amino Acids, 2010, 38, 189-197.

11 J. Mielcarek, D. M. Nowak, A. Pajzderska, B. Peplinska and J. Wasicki, A Hybrid Method for Estimation of Molecular Dynamics of Diazepam-Density Functional Theory Combined with NMR and FT-IR spectroscopy, Int. J. Pharm. Res. Scholars, 2011, 404, 19-26.

12 J. Wạsicki, D. P. Kozlenko, S. E. Pankov, P. Bilski, A. Pajzderska, B. C. Hancock, A. Medek, W. Nawrocik and B. N. Savenko, NMR Search for Polymorphic Phase Transformations in Chlorpropamide form-A at High Pressures, J. Pharm. Sci., 2009, 98, 1426-1437.

13 A. Pajzderska, D. M. Chudoba, J. Mielcarek and J. Wasicki, Calorimetric, FTIR and ${ }^{1} \mathrm{H}$ NMR Measurements in Combination with DFT Calculations for Monitoring Solid-
State Changes of Dynamics of Sibutramine Hydrochloride, J. Pharm. Sci., 2012, 101, 3799-3810.

14 E. R. Andrew, B. Peplinska and M. Kempka, Molecular Dynamics in Solid l-adrenaline by Proton NMR, Solid State Nucl. Magn. Reson., 1998, 10, 117-121.

15 C. Smuda, S. Busch, R. Schellenberg and T. Unruh, Methyl Group Dynamics in Polycrystalline and Liquid Ubiquinone Qo Studied by Neutron Scattering, J. Phys. Chem. B, 2009, 113, 916-922.

16 A. Pajzderska, M. Jarek, J. Mielcarek and J. Wasicki, Analysis of the Distribution of Energy Barriers in Amorphous Diazepam on the Basis of Computationally Supported NMR Relaxation Data, J. Phys. Chem. B, 2016, 120, 1072310728.

17 I. T. Todorov, W. Smith, K. Trachenko and M. T. Dove, DL_POLY_3: New Dimensions In Molecular Dynamics Simulations via Massive Parallelism, J. Mater. Chem., 2006, 16, 1911-1918.

18 A. Camerman and N. Camerman, Stereochemical Basis of Anticonvulsant Drug Action. II. Molecular Structure of Diazepamu, J. Am. Chem. Soc., 1972, 94, 268-272.

19 Materials Studio Modelling Environment, Version 6.5, Accelrys, Inc., San Diego, CA, 2009.

20 H. J. C. Berendsen, J. P. M. Postma, W. van Gunsteren, A. DiNola and J. R. Haak, Molecular Dynamics with Coupling to an External Bath, J. Chem. Phys., 1984, 81, 3684. 21 P. Dauber-Osguthorpe, V. A. Roberts, D. J. Osguthorpe, J. Wolff, M. Genest and A. T. Hagler, Structure and energetics of ligand binding to proteins: Escherichia coli dihydrofolate reductase-trimethoprim, a drug-receptor system, Proteins: Struct., Funct., Genet., 1988, 4, 31-47.

22 K. Hinsen, E. Pellegrini, S. Stachura and G. R. Kneller, nMoldyn 3: Using Task Farming for a Parallel Spectroscopy-oriented Analysis of Molecular Dynamics Simulations, J. Comput. Chem., 2012, 33, 2043-2048.

23 R. T. Azuah, L. R. Kneller, Y. Qiu, P. L. W. Tregenna-Piggott, C. M. Brown, J. R. D. Copley and R. M. Dimeo, DAVE: A Comprehensive Software Suite for the Reduction, Visualization, and Analysis of Low Energy Neutron Spectroscopic Data, J. Res. Natl. Inst. Stand. Technol., 2009, 114, 341-358.

24 D. Richard, M. Ferrand and G. J. Kearley, Analysis and Visualisation of Neutron-Scattering Data, J. Neutron Res., 1996, 4, 33-39.

25 M. Bee, Quasielastic Neutron Scattering: Principles and Applications in Solid State Chemistry, Biology, and Material Science, Adam Hilger, Bristol, Philadelphia, 1988.

26 A. Chahid, A. Alegría and J. Colmenero, Methyl Group Dynamics in Poly(vinyl methyl ether). A Rotation Rate Distribution Model, Macromolecules, 1994, 27, 3282-3288.

27 A. C. Genix, A. Arbe, J. Colmenero, J. Wuttke and D. Richter, Neutron Scattering and X-ray Investigation of the Structure and Dynamics of Poly(ethyl methacrylate), Macromolecules, 2012, 45, 2522-2536.

28 F. Alvarez, A. Alegría and J. Colmenero, Origin of the Distribution of Potential Barriers for Methyl Group Dynamics in Glassy Polymers: A Molecular Dynamics 
Simulation in Polyisoprene, Macromolecules, 2000, 33, 80778084.

29 A. J. Moreno, A. Alegria and J. Colmenero, Methyl Group Dynamics in Glassy Toluene: A Neutron Scattering Study, J. Chem. Phys., 2001, 115, 8958-8966.

30 A. J. Moreno, A. Alegria, J. Colmenero and B. Frick, Methyl Group Dynamics from Tunneling to Hopping in
$\mathrm{NaCH}_{3} \mathrm{CO}_{2} \cdot 3 \mathrm{H}_{2} \mathrm{O}$ : Comparison Between a Crystal and its Glassy Counterpart, Phys. Rev. B: Condens. Matter Mater. Phys., 2002, 65, 134202.

31 R. Zorn, M. Mayorova, D. Richter and B. Frick, Inelastic Neutron Scattering Study of a Glass-Forming Liquid in Soft confinement, Soft Matter, 2008, 4, 522-533. 